Supplementary Information for:

\title{
Ab Initio Flexible Force Field for Metal-Organic Frameworks Using Dummy Model Coordination Bonds
}

Sudi Jawahery, Nakul Rampal, Seyed Mohamad Moosavi, Matthew

Witman, and Berend Smit 
Residual Sum of Squares for Metal-Oxygen Forces

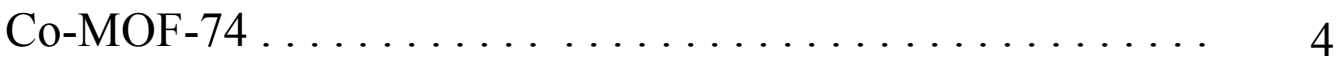

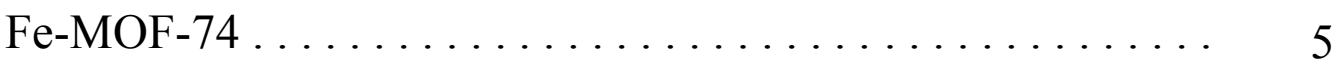

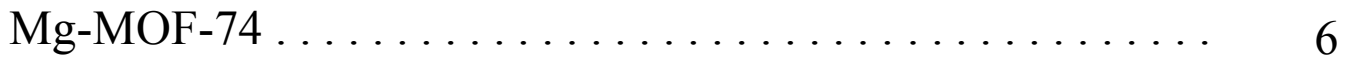

Mn-MOF-74 .................... 7

Ni-MOF-74 ................... 8

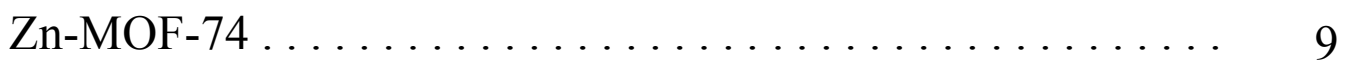

Total Metal-Oxygen Forces

Metal-Ob....................... 10

Metal-Oc ........................ 11

Simulated Radial Distribution Function at 298 K . . . . . . . . . 12

Free Energy of Deformation with Duarte et al.-adapted Force Field. . 13

Fitted Force Field Parameters

Metal-Oxygen . . . . . . . . . . . 15

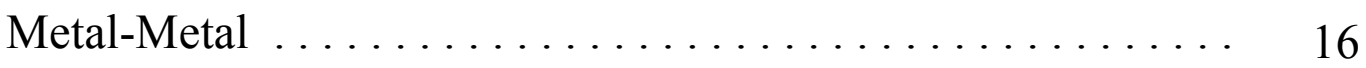

Configurations for Fitting $\ldots \ldots \ldots \ldots \ldots \ldots \ldots \ldots \ldots \ldots \ldots$

M-MOF-274 Ligand $\ldots \ldots \ldots \ldots \ldots \ldots \ldots \ldots \ldots \ldots \ldots \ldots \ldots \ldots \ldots \ldots \ldots \ldots \ldots$

Result Comparison from Different $m$-DFT Calculations. . . . . . . 21 


\section{Residual Sum of Squares for Metal-Oxygen Forces}

The next pages show weighted RSS plots for the different metal-oxygen interactions. As in Figure 3 of the main text, the peaks in data of each panel of reflect the correlation of the weighted RSS to the weighting function, which is plotted as a black dashed line. The Duarte et al.-adapted values are shown in red, while the fitted force field values are shown in blue. In the metal-oxygen graphs, the peaks are difficult to distinguish due to the large divergence of the Duarte et al.-adapted parameters at low distances. The Duarte et al.-adapted forces are frequently so inaccurate that the RSS values are beyond the boundaries of the graphs. The right-side panels are zoomed-in to allow the fitted force field data to be better visualized. 

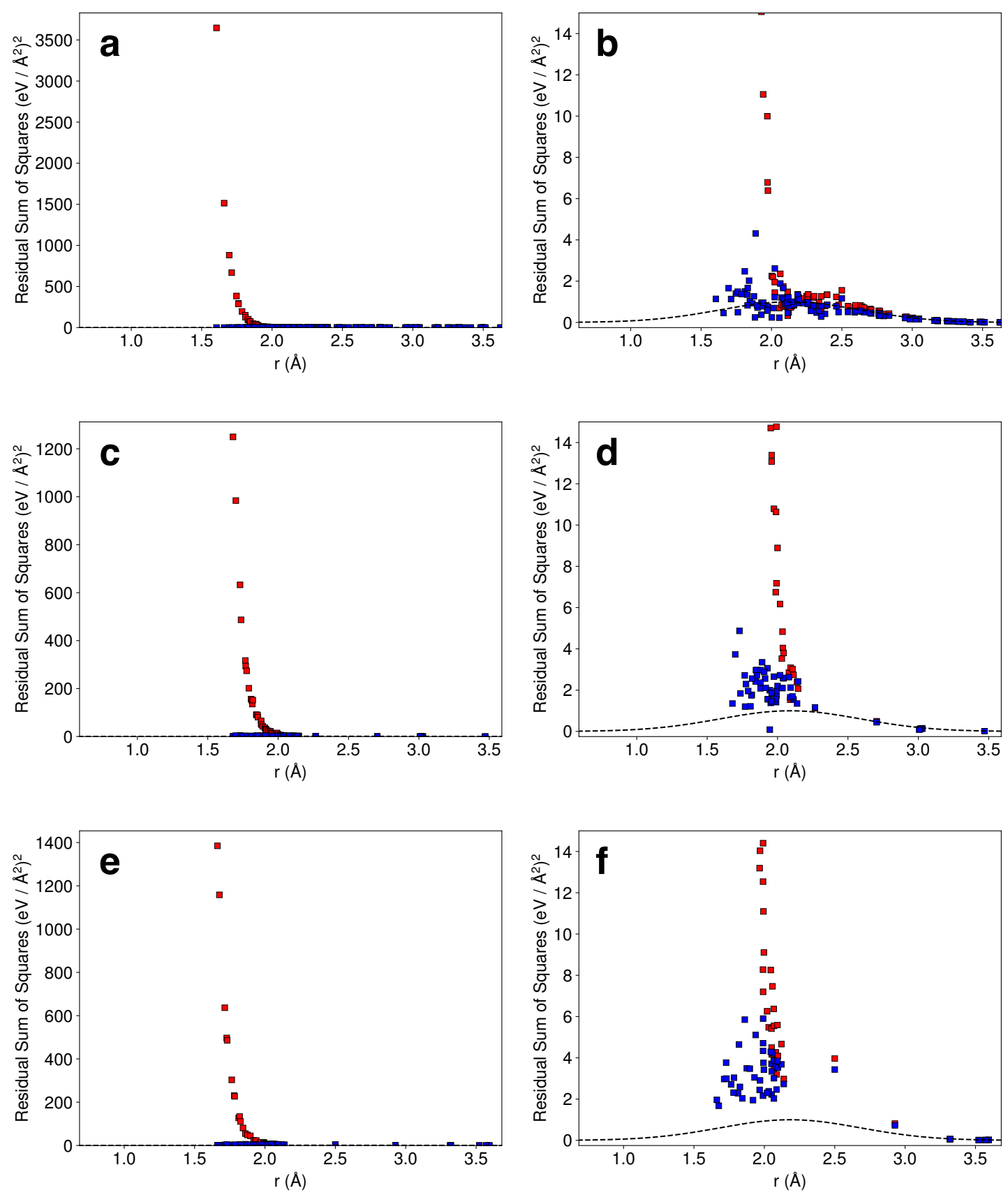

Supplementary Figure 1: Co-MOF-74: The weighted residual sum of squares (RSS) between the force field force vectors and the DFT force vectors resulting from CoOxygen interactions. The Duarte et al.-adapted RSS values are plotted as red points, while the fitted RSS values are plotted as blue points. Panels (a) and (b) show Co-Oa RSS values; panels (c) and (d) show Co-Ob RSS values; panels (e) and (f) show Co-Oc RSS values. The black dashed curve is the Gaussian weighting function with standard deviation $0.5 \AA$. 

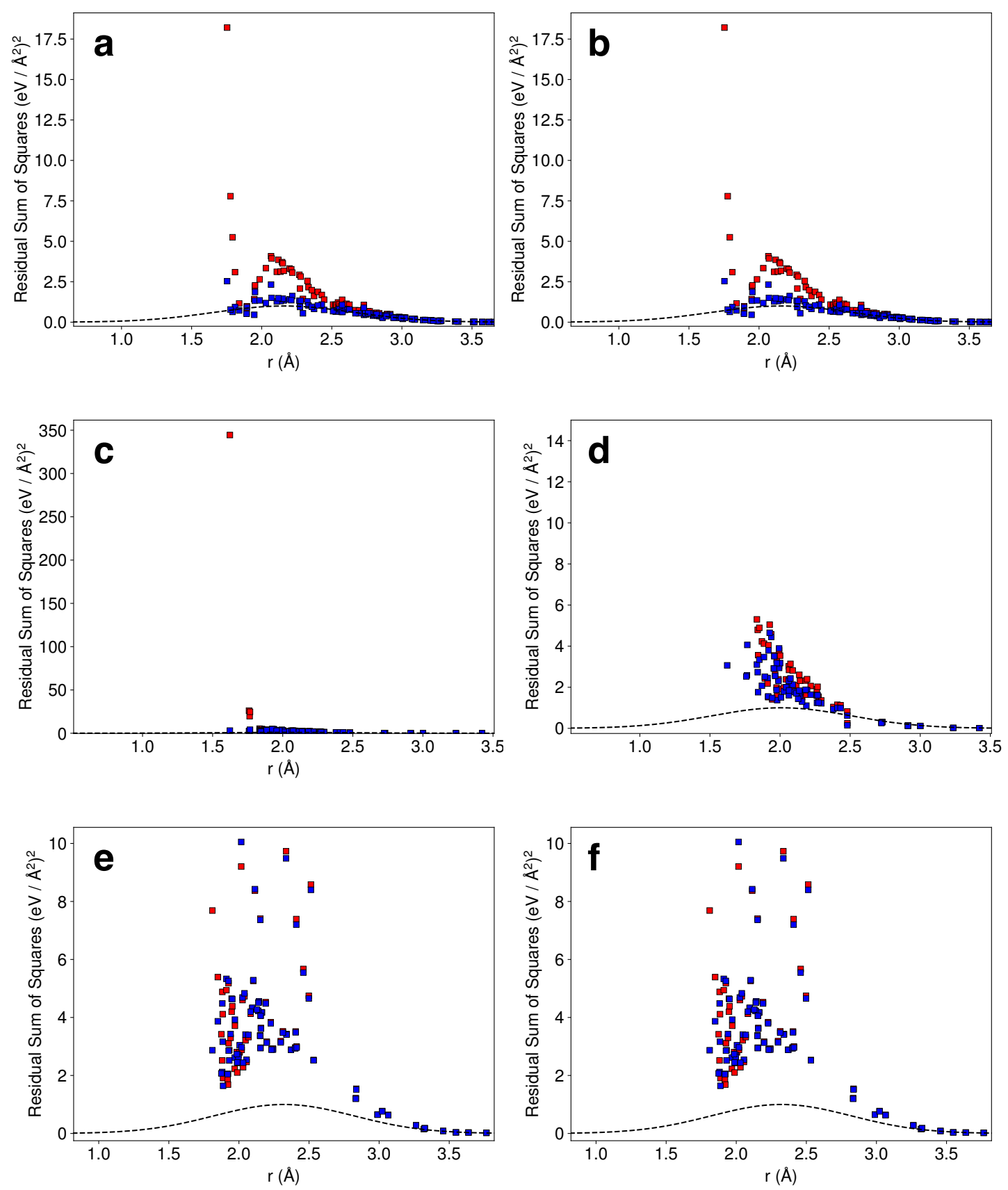

Supplementary Figure 2: Fe-MOF-74: The weighted residual sum of squares (RSS) between the force field force vectors and the DFT force vectors resulting from FeOxygen interactions. The Duarte et al.-adapted RSS values are plotted as red points, while the fitted RSS values are plotted as blue points. Panels (a) and (b) show Fe-Oa RSS values; panels (c) and (d) show Fe-Ob RSS values; panels (e) and (f) show Fe-Oc RSS values. The black dashed curve is the Gaussian weighting function with standard deviation $0.5 \AA$. 

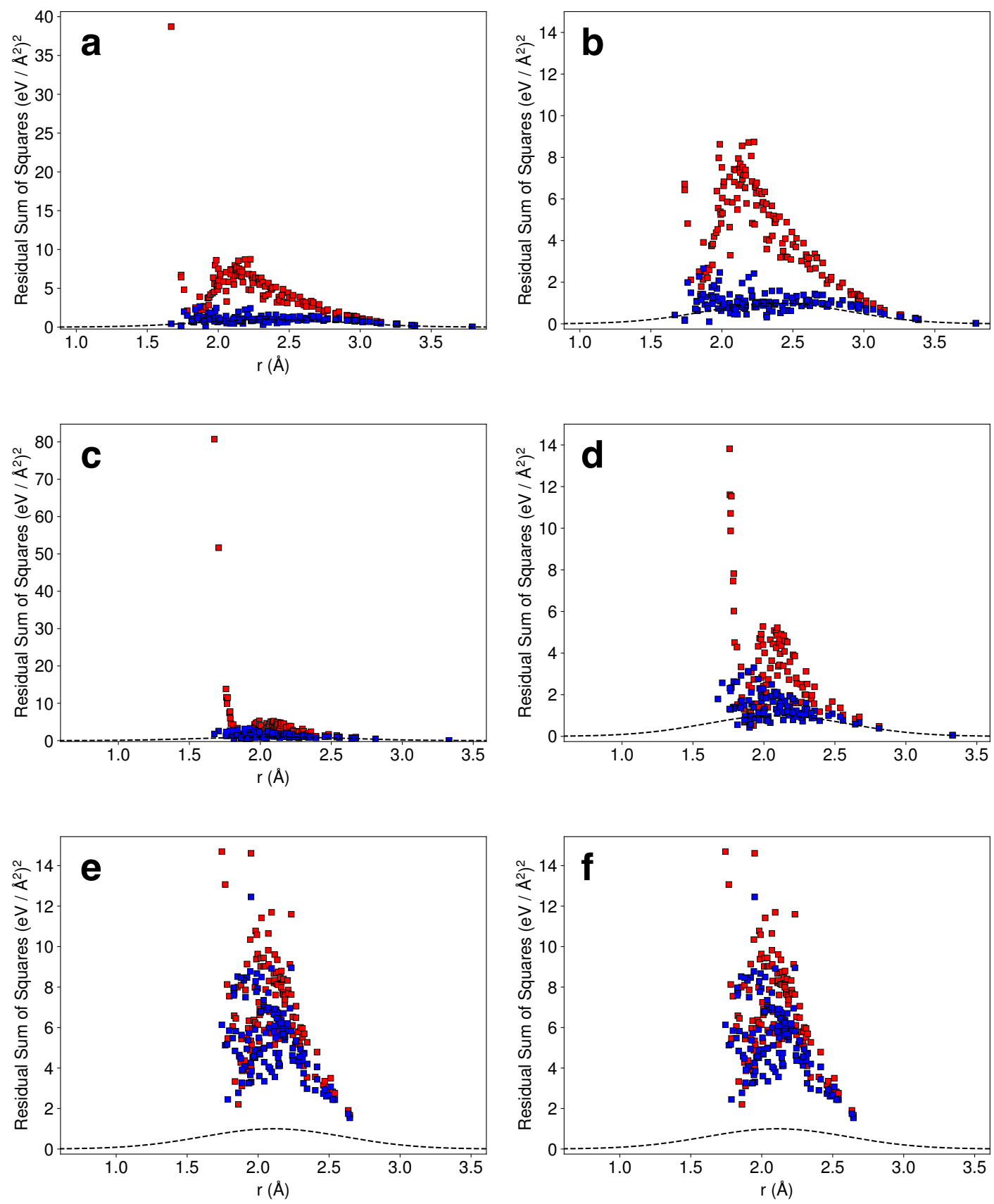

Supplementary Figure 3: Mg-MOF-74: The weighted residual sum of squares (RSS) between the force field force vectors and the DFT force vectors resulting from MgOxygen interactions. The Duarte et al.-adapted RSS values are plotted as red points, while the fitted RSS values are plotted as blue points. Panels (a) and (b) show Mg-Oa RSS values; panels (c) and (d) show Mg-Ob RSS values; panels (e) and (f) show Mg-Oc RSS values. The black dashed curve is the Gaussian weighting function with standard deviation $0.5 \AA$. 

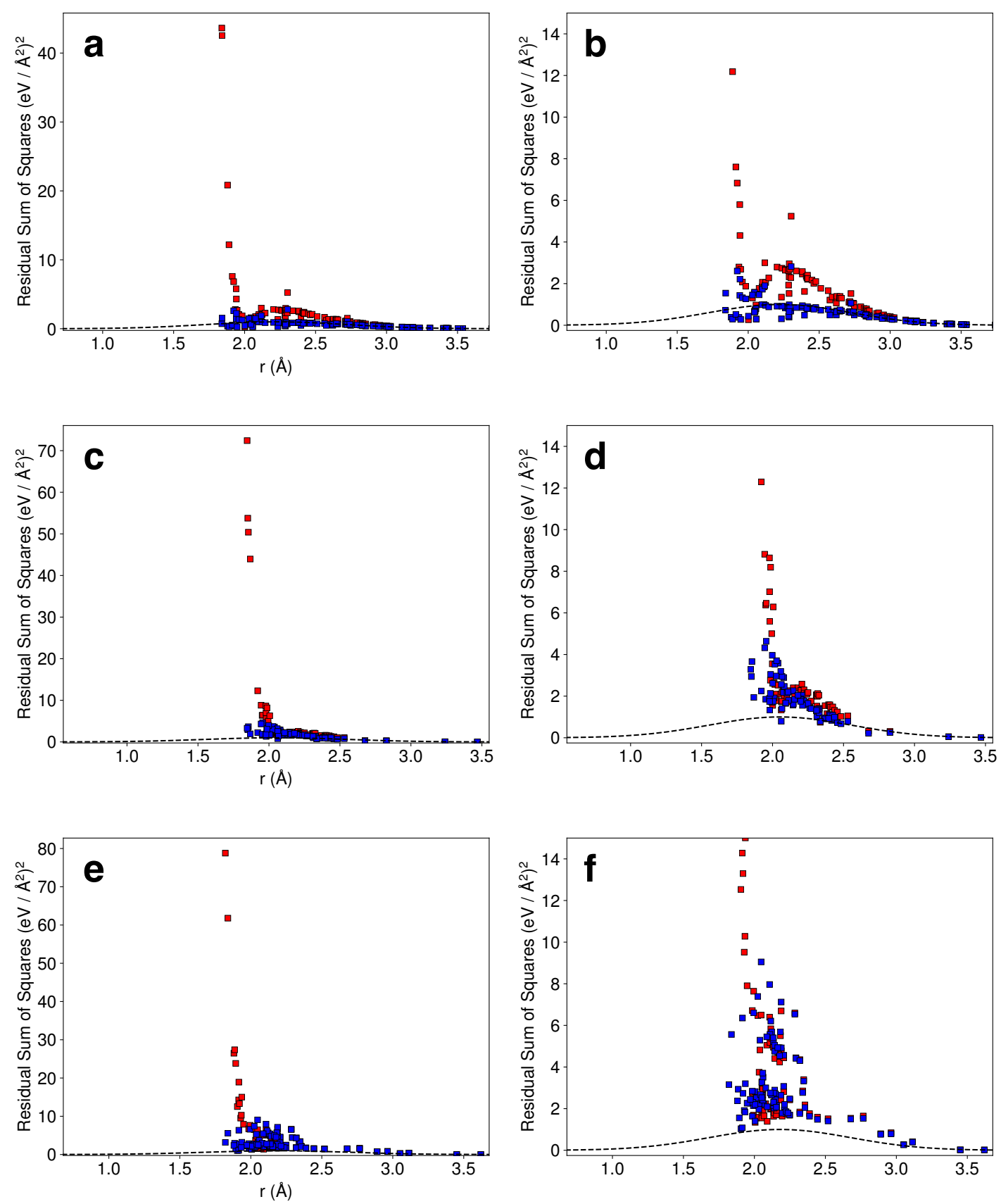

Supplementary Figure 4: Mn-MOF-74: The weighted residual sum of squares (RSS) between the force field force vectors and the DFT force vectors resulting from MnOxygen interactions. The Duarte et al.-adapted RSS values are plotted as red points, while the fitted RSS values are plotted as blue points. Panels (a) and (b) show Mn-Oa RSS values; panels (c) and (d) show Mn-Ob RSS values; panels (e) and (f) show Mn-Oc RSS values. The black dashed curve is the Gaussian weighting function with standard deviation $0.5 \AA$. 

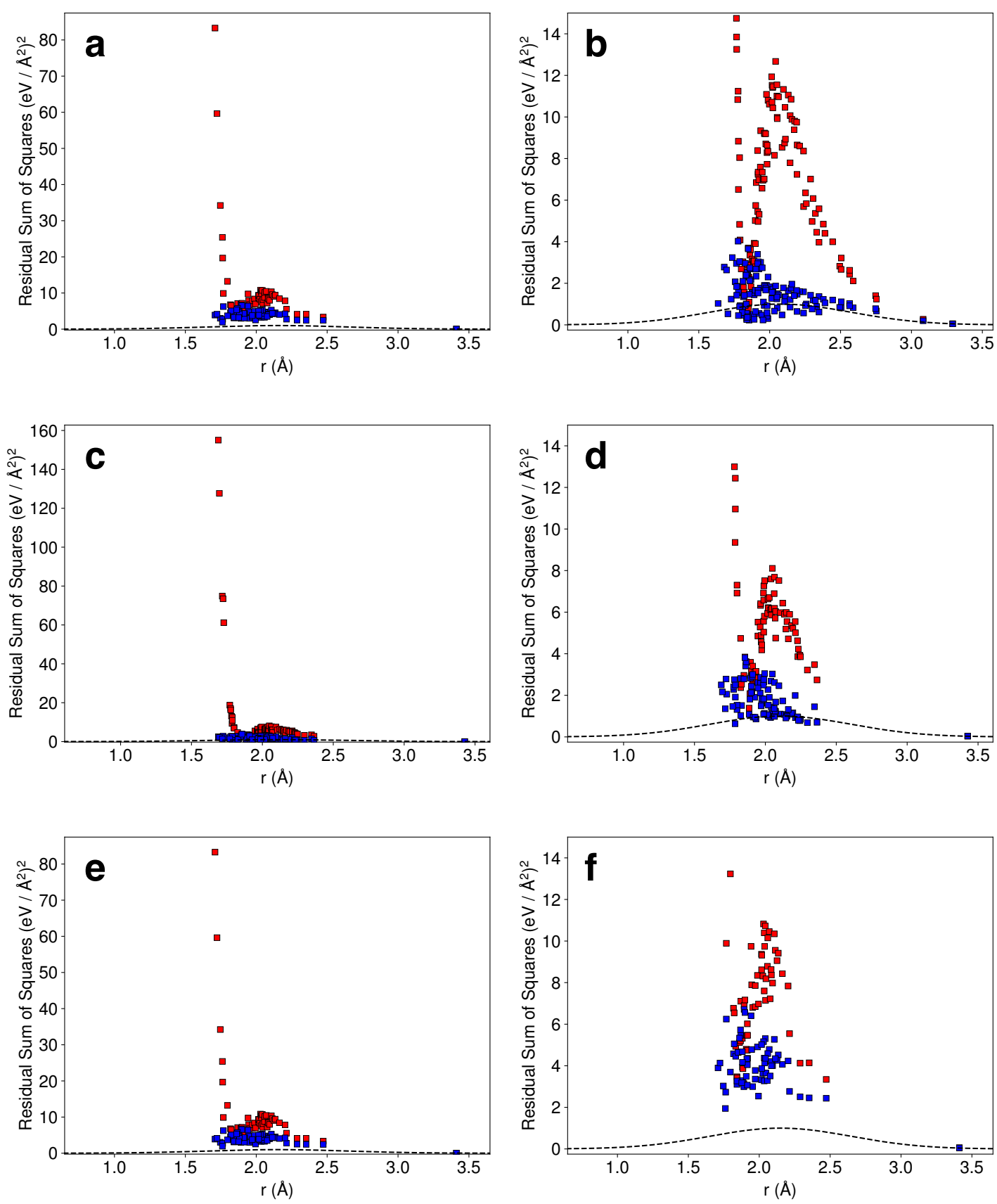

Supplementary Figure 5: Ni-MOF-74: The weighted residual sum of squares (RSS) between the force field force vectors and the DFT force vectors resulting from NiOxygen interactions. The Duarte et al.-adapted RSS values are plotted as red points, while the fitted RSS values are plotted as blue points. Panels (a) and (b) show Ni-Oa RSS values; panels (c) and (d) show Ni-Ob RSS values; panels (e) and (f) show Ni-Oc RSS values. The black dashed curve is the Gaussian weighting function with standard deviation $0.5 \AA$. 

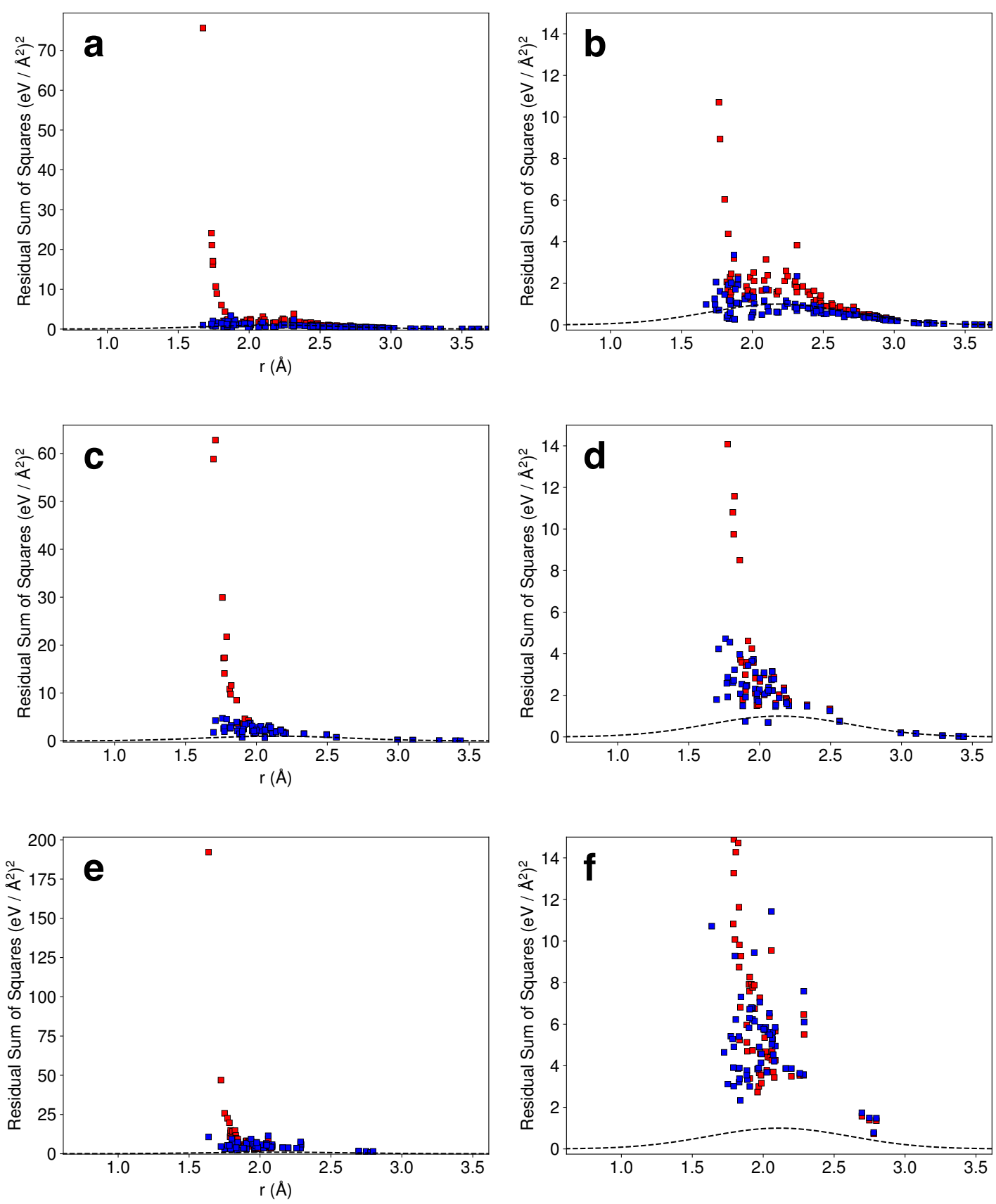

Supplementary Figure 6: Zn-MOF-74: The weighted residual sum of squares (RSS) between the force field force vectors and the DFT force vectors resulting from ZnOxygen interactions. The Duarte et al.-adapted RSS values are plotted as red points, while the fitted RSS values are plotted as blue points. Panels (a) and (b) show Zn-Oa RSS values; panels (c) and (d) show Zn-Ob RSS values; panels (e) and (f) show Zn-Oc RSS values. The black dashed curve is the Gaussian weighting function with standard deviation $0.5 \AA$. 


\section{Total Metal-Oxygen Forces}
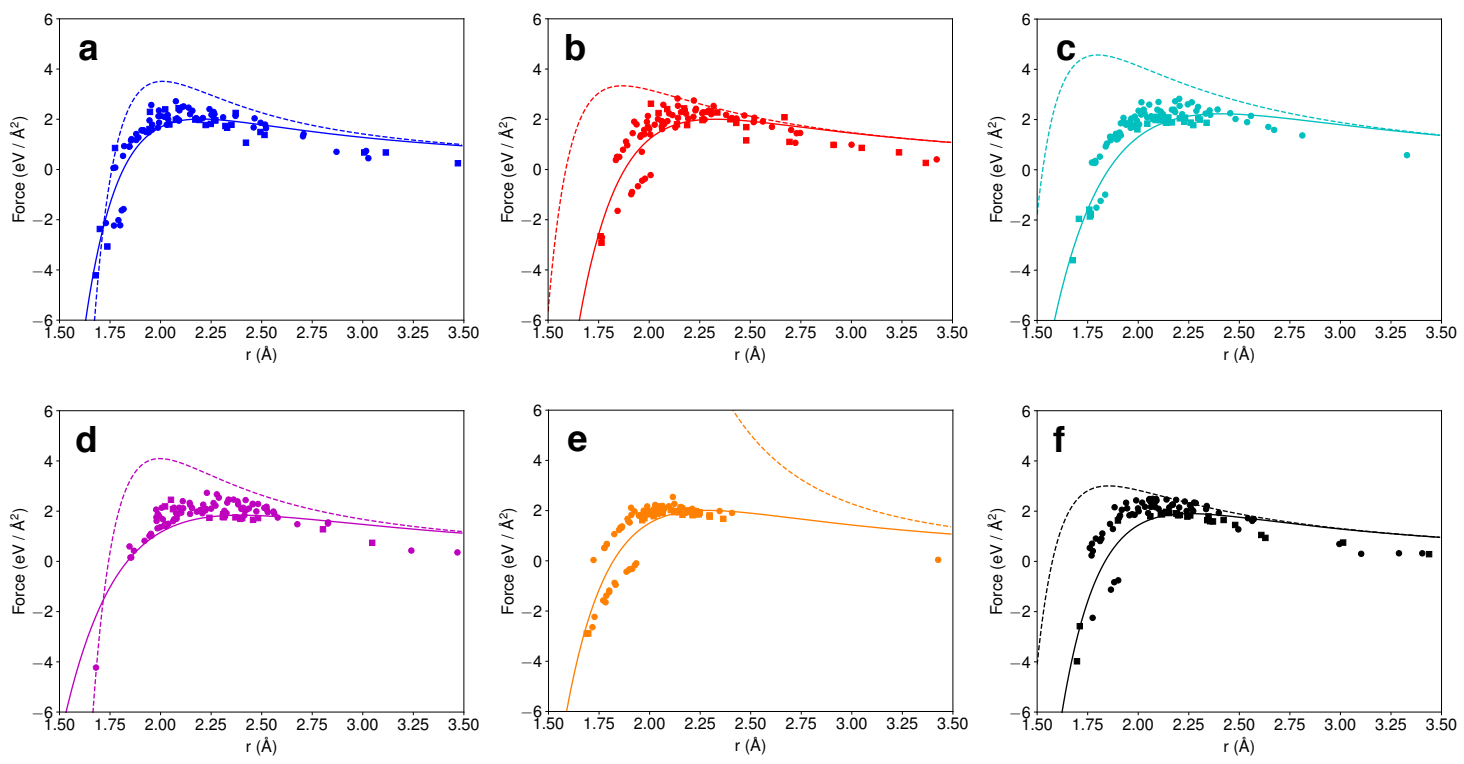

Supplementary Figure 7: Panels (a)-(f) show how the estimated metal- $\mathrm{O}_{\mathrm{b}}$ forces from $a b$ initio calculations are the reproduced by the parameterized Born-Mayer-Huggins + Coulomb potential. Panels (a), (b), (c), (d), (e), and (f) show data for Co-, Fe-, Mg-, Mn-,

$\mathrm{Ni}$-, and Zn-MOF-74, respectively. The squares each represent a different system configuration used for a DFT calculation, and the lines represent the fitted force functions as we have parameterized them in this work. 

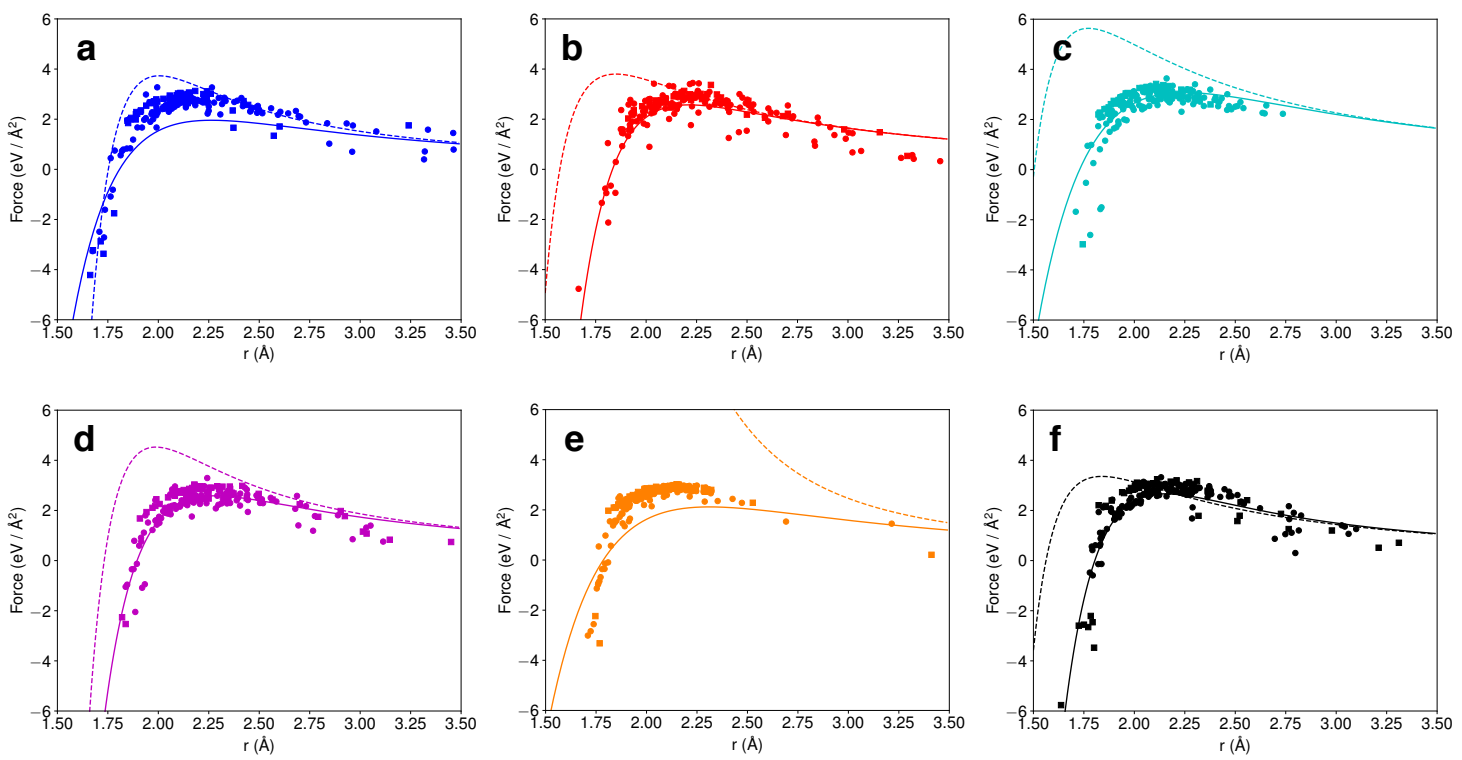

Supplementary Figure 8: Panels (a)-(f) show how the estimated metal- $\mathrm{O}_{c}$ forces from $a b$ initio calculations are the reproduced by the parameterized Born-Mayer-Huggins + Coulomb potential. Panels (a), (b), (c), (d), (e), and (f) show data for Co-, Fe-, Mg-, Mn-,

$\mathrm{Ni}$-, and Zn-MOF-74, respectively. The squares each represent a different system configuration used for a DFT calculation, and the lines represent the fitted force functions as we have parameterized them in this work. 

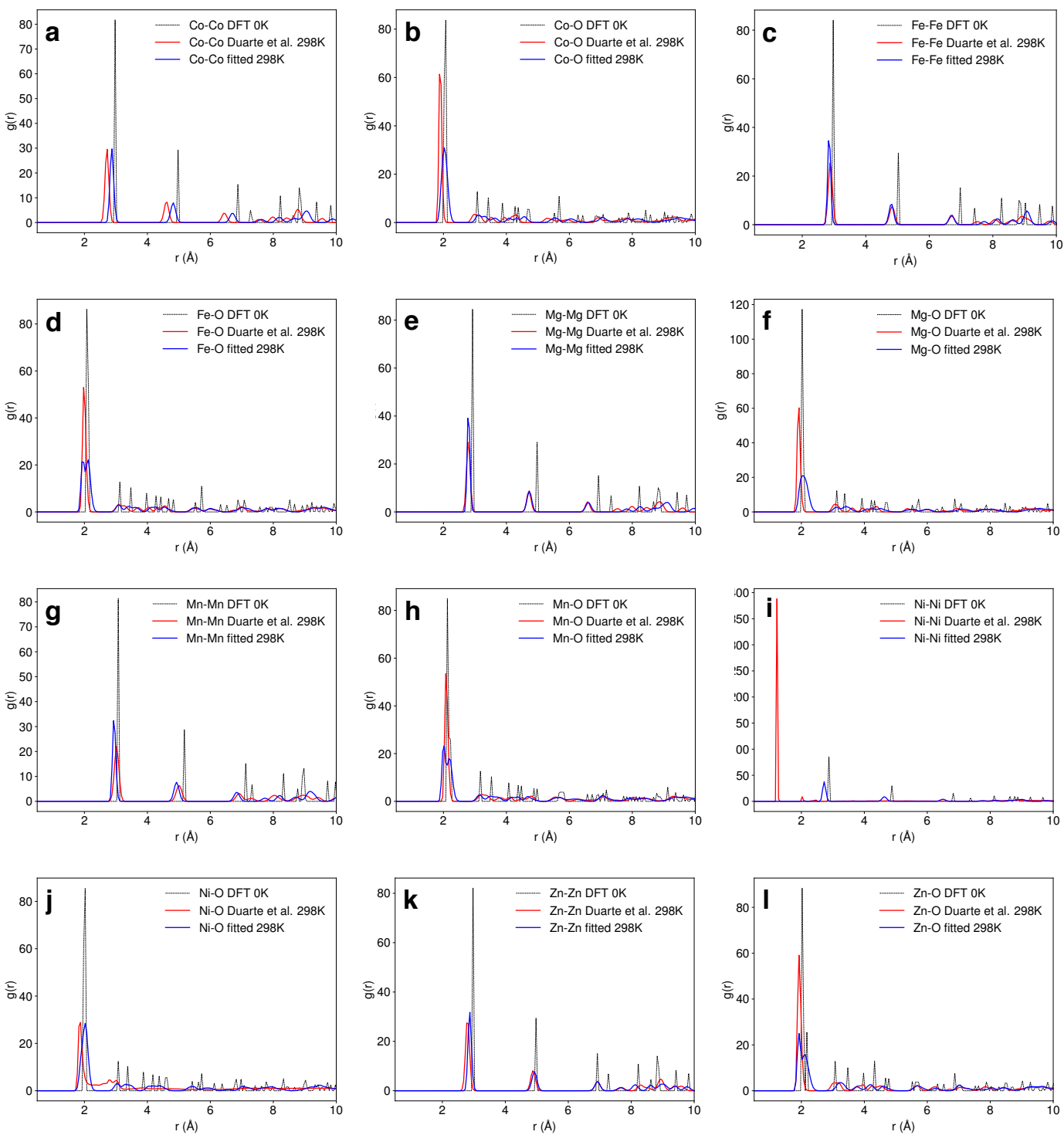

Supplementary Figure 9: The metal-metal radial distribution functions (RDFs) resulting from force field molecular simulations at $298 \mathrm{~K}$ of Co-, Fe-, Mg-, Mn-, Ni- and Zn-MOF74 are shown in panels (a), (c), (e), (g), (i) and (k), respectively. The metal-oxygen RDFs resulting from force field molecular simulations at $298 \mathrm{~K}$ of Co-, Fe-, $\mathrm{Mg}-, \mathrm{Mn}-, \mathrm{Ni}-$ and Zn-MOF-74 are shown in panels (b), (d), (f), (h), (j) and (l), respectively. The fitted force field RDFs are plotted in blue, while the Duarte et al.-adapted force field RDFs are plotted in red. The RDF calculated from the DFT optimized structure at $0 \mathrm{~K}$ is plotted as a black dashed line and is included to facilitate comparisons between the predictions. 


\section{Free Energy of Deformation with Duarte et al.-adapted Force}

Field

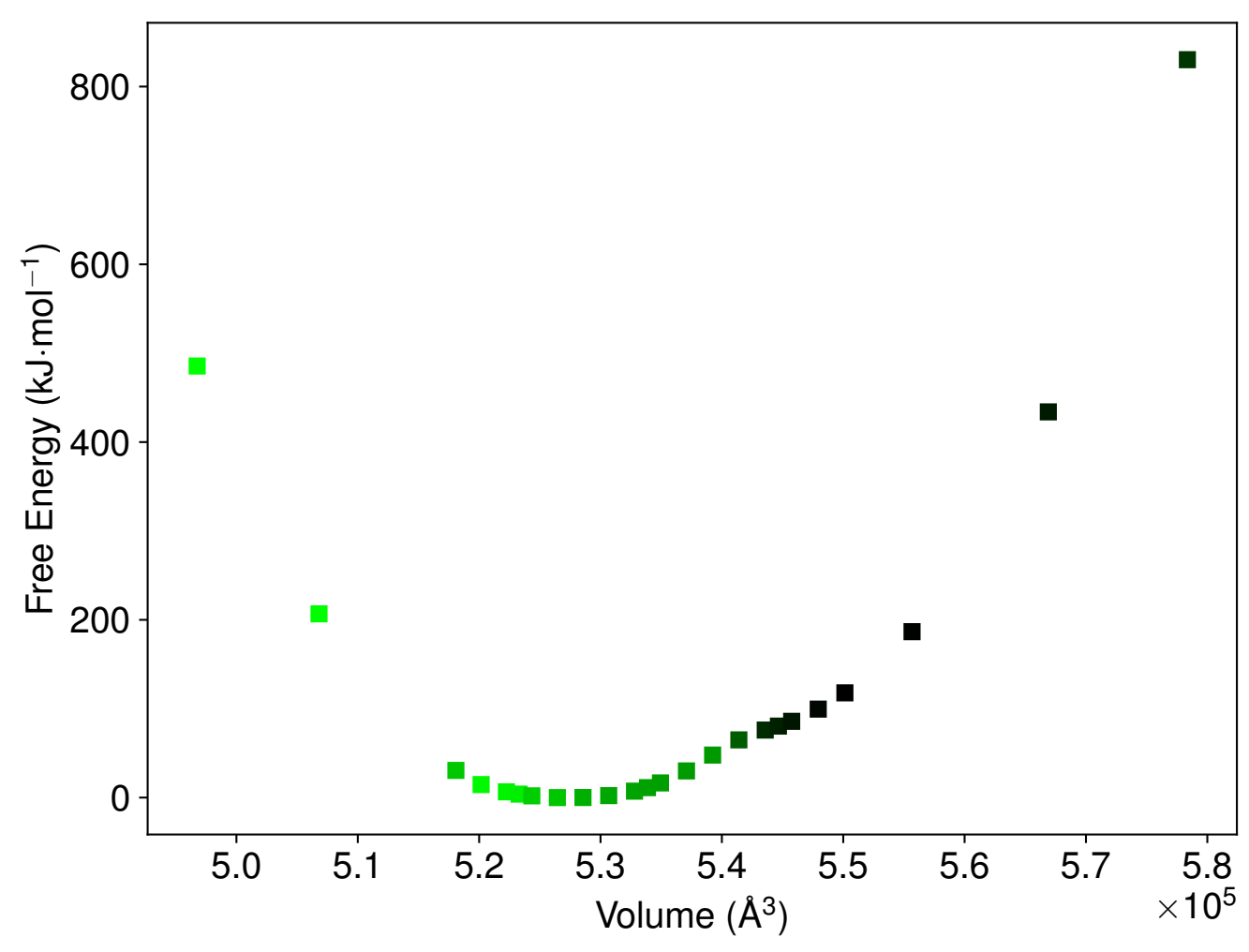

Supplementary Figure 10: The free energy profile as a function of volume for the $\mathrm{Mg}$ MOF-574 system with 486 Argon per channel, modeled using the Duarte et al.-adapted force field. In this free energy profile, there is only one free energy minimum at the deformed structure volume. 


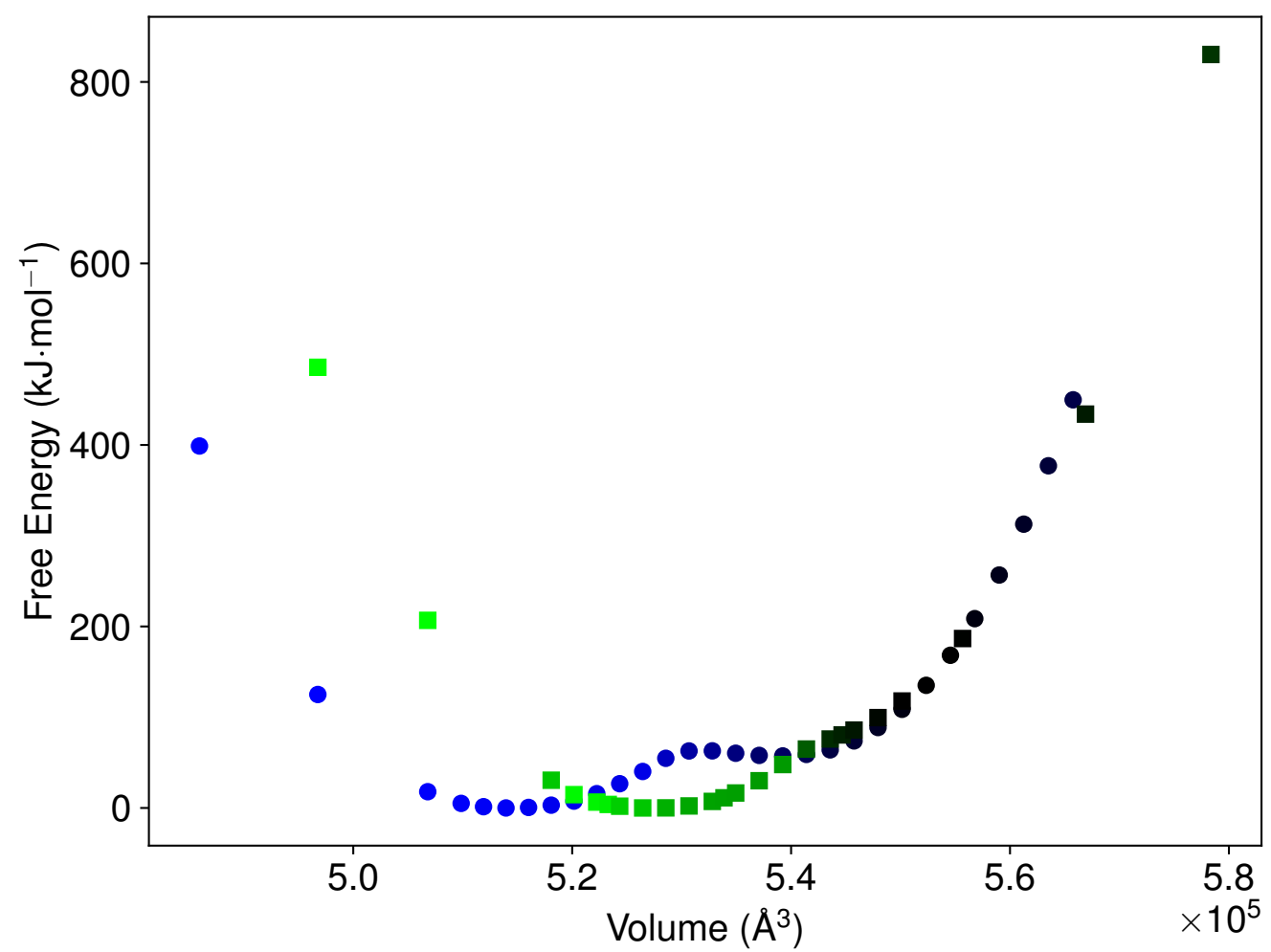

Supplementary Figure 11: The free energy profiles as a function of volume for the $\mathrm{Mg}$ MOF-574 system with 486 Argon per channel, modeled using both the Duarte et al.adapted force field (black and green squares) and the force field developed in this work (blue and black circles). This plot is an overlay of the data from Figure 7 and SI Figure 10 and highlights the differences in the free energy features: 1) the parameterized force field shows two free energy minima, while the Duarte et al.-adapted force field shows one and 2) the minima predicted by the different models occur at different volumes. 


\section{Fitted Force Field Parameters}

Table 1: Co-Oxygen parameters for Co-MOF-74

\begin{tabular}{|c|c|c|c|}
\hline & $\mathbf{A}(\mathbf{k c a l} / \mathbf{m o l})$ & $\mathbf{B}\left(\AA^{-1}\right)$ & $\mathbf{C}\left(\mathbf{k c a l} \cdot \AA^{\mathbf{6}}\right) / \mathbf{m o l}$ \\
\hline $\mathbf{C o - O a}$ & 99.49 & 4.33 & 0.0 \\
\hline $\mathbf{C o - O b}$ & 87.04 & 5.83 & 0.0 \\
\hline $\mathbf{C o - O c}$ & 78.49 & 4.61 & 0.0 \\
\hline
\end{tabular}

Table 2: Fe-Oxygen parameters for Fe-MOF-74

\begin{tabular}{|c|c|c|c|}
\hline & $\mathbf{A}(\mathbf{k c a l} / \mathbf{m o l})$ & $\mathbf{B}\left(\AA^{-1}\right)$ & $\mathbf{C}\left(\mathbf{k c a l} \cdot \AA^{6}\right) / \mathbf{m o l}$ \\
\hline $\mathbf{F e}-\mathbf{O a}$ & 119.77 & 4.17 & 0.0 \\
\hline $\mathbf{F e}-O b$ & 117.04 & 4.70 & 98.29 \\
\hline $\mathbf{F e}-O c$ & 127.79 & 5.92 & 46.74 \\
\hline
\end{tabular}

Table 3: Mg-Oxygen parameters for Mg-MOF-74

\begin{tabular}{|c|c|c|c|}
\hline & $\mathbf{A}(\mathbf{k c a l} / \mathbf{m o l})$ & $\mathbf{B}\left(\AA^{-1}\right)$ & $\mathbf{C}\left(\mathbf{k c a l} \cdot \AA^{\mathbf{6}}\right) / \mathbf{m o l}$ \\
\hline Mg-Oa & 137.31 & 2.5 & 128.62 \\
\hline Mg-Ob & 111.86 & 3.55 & 0.0 \\
\hline Mg-Oc & 134.87 & 3.49 & 0.0 \\
\hline
\end{tabular}


Table 4: Mn-Oxygen parameters for Mn-MOF-74

\begin{tabular}{|c|c|c|c|}
\hline & $\mathbf{A}(\mathbf{k c a l} / \mathbf{m o l})$ & $\mathbf{B}\left(\AA^{-1}\right)$ & $\mathbf{C}\left(\mathbf{k c a l} \cdot \AA^{\mathbf{6}}\right) / \mathbf{m o l}$ \\
\hline Mn-Oa & 131.25 & 3.61 & 0.0 \\
\hline Mn-Ob & 87.72 & 3.51 & 0.0 \\
\hline Mn-Oc & 182.10 & 5.88 & 72.41 \\
\hline
\end{tabular}

Table 5: Ni-Oxygen parameters for Ni-MOF-74

\begin{tabular}{|c|c|c|c|}
\hline & $\mathbf{A}(\mathbf{k c a l} / \mathbf{m o l})$ & $\mathbf{B}\left(\AA^{-1}\right)$ & $\mathbf{C}\left(\mathbf{k c a l} \cdot \AA^{\mathbf{6}}\right) / \mathbf{m o l}$ \\
\hline Ni-Oa & 104.20 & 3.89 & 0.0 \\
\hline Ni-Ob & 85.24 & 4.51 & 0.0 \\
\hline Ni-Oc & 82.71 & 3.79 & 0.0 \\
\hline
\end{tabular}

Table 6: Zn-Oxygen parameters for Zn-MOF-74

\begin{tabular}{|c|c|c|c|}
\hline & $\mathbf{A}(\mathbf{k c a l} / \mathbf{m o l})$ & $\mathbf{B}\left(\AA^{-\mathbf{1}}\right)$ & $\mathbf{C}\left(\mathbf{k c a l} \cdot \AA^{\mathbf{6}}\right) / \mathbf{m o l}$ \\
\hline Zn-Oa & 102.84 & 3.99 & 0.0 \\
\hline Zn-Ob & 87.93 & 5.18 & 0.0 \\
\hline Zn-Oc & 150.96 & 5.83 & 603.83 \\
\hline
\end{tabular}


Table 7: Metal-Metal parameters for all M-MOF-74 analogs

\begin{tabular}{|c|c|c|}
\hline & E $(\mathbf{k c a l} / \mathbf{m o l})$ & $\boldsymbol{\sigma}(\AA)$ \\
\hline Co-Co & 19.66 & 2.48 \\
\hline Fe-Fe & 29.60 & 2.49 \\
\hline Mg-Mg & 61.09 & 2.41 \\
\hline Mn-Mn & 28.96 & 2.56 \\
\hline Ni-Ni & 36.03 & 2.32 \\
\hline Zn-Zn & 23.96 & 2.47 \\
\hline
\end{tabular}




\section{Configurations for Fitting}

Pairwise force fitting was not possible for the forces generated from all configurations because upon subtracting the electrostatic contributions, the components of the remaining contributions occasionally were not all aligned with either an attractive or repulsive force

vector. For example, for a given configuration being tested the $x$-component $\left(F_{j, x}^{\prime \prime}\right)$ of the estimated metal-oxygen dispersion force vector may point in the direction of an attractive metal-oxygen vector, while the $y$-component $\left(F_{j, y}^{\prime \prime}\right)$ points in the opposite direction. This problem could have two possible sources: 1. the assumption we make that the metaloxygen interaction is purely pairwise is not valid for this case, and/or 2. the electrostatic interactions for this case are not accurately captured by the Mercado et al. charges. This case was impossible to fit, and such data were therefore excluded.

We investigated the potential impact of these excluded data points. The blue data shown on Fig. 11a is identical to Fig. 4a of the main text. The cyan and black data points represent the excluded data points, and span the same set of configurations. The magnitudes of the force plotted were calculate differently for the cyan and black sets the cyan magnitudes were calculated using all components of the force (included nonaligned components), while black magnitudes were calculated using only the components that were aligned consistently with the expected largest force component (the largest component of the distance vector should correspond with the largest force component).

For most of the excluded data, the black circles and cyan stars overlap. This indicates that much of this data was excluded due to components that contribute only slightly to the total magnitude of the force. We evaluated the impact of excluding configurations by refitting the forces while reincluding the configurations where the misaligned force components make up less than $10 \%$ of the total force magnitude (the majority of cases). As shown in Table 8, the impact of excluding these configurations is minimal, as all of the force field parameters change by less than $1 \%$. 

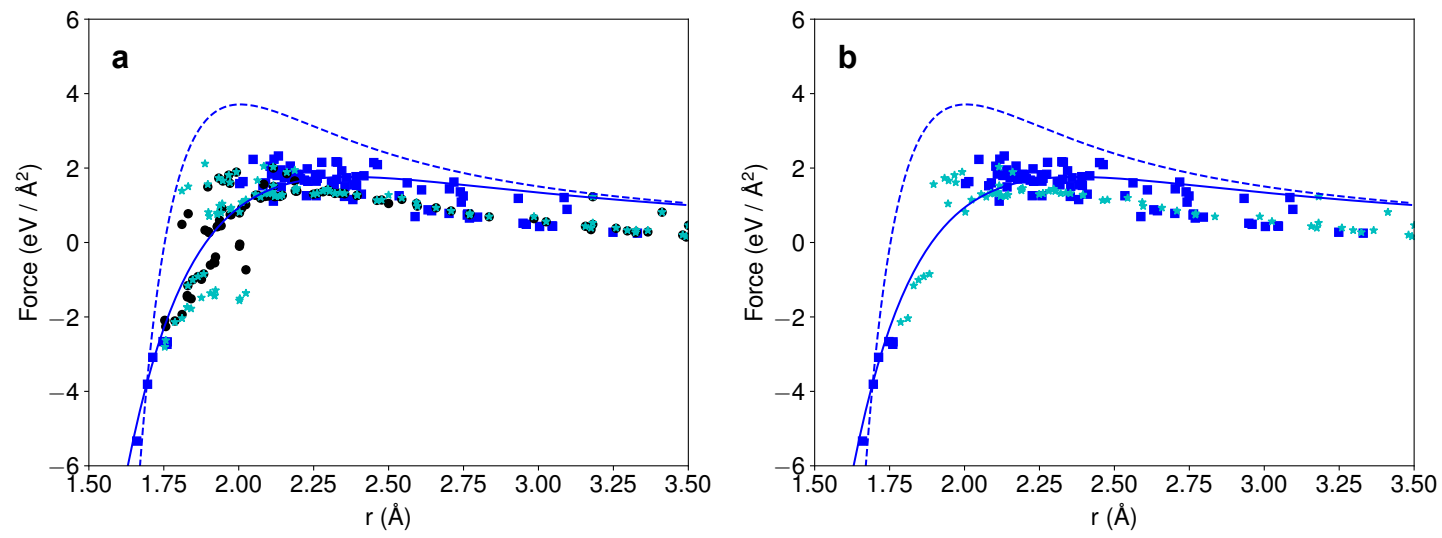

Supplementary Figure 12: Force data for the Co-Oa pair in Co-MOF-74. The

blue solid and dashed lines reflect fitted and Duarte et al.-adapted force calculations, respectively. In both (a) and (b) blue squares represent DFT force configurations included in the fitting. In (a) black circles represent the magnitude of the aligned components of forces excluded from the fitting, and cyan stars represent the full magnitude of forces excluded from the fitting. In (b) cyan stars represent additional data included in the test of

Table 8: Co-Oxygen parameters for Co-MOF-74 showing a comparison of force field parameters between two cases: 1) no non-aligned force configurations are included in the fitting procedure, 2) non-aligned forces are included in the fitting procedure provided the non-aligned components contribute to less than $10 \%$ of the total force magnitude.

\begin{tabular}{|c|c|c|c|}
\hline & $\mathbf{A}(\mathbf{k c a l} / \mathbf{m o l})$ & $\mathbf{B}\left(\AA^{-1}\right)$ & $\mathbf{C ~}\left(\mathbf{k c a l} \cdot \AA^{\mathbf{6}}\right) / \mathbf{m o l}$ \\
\hline Co-Oa, fitted & 99.49 & 4.33 & 0.0 \\
\hline $\begin{array}{c}\text { Co-Oa, additional } \\
\text { data }\end{array}$ & 99.62 & 4.32 & 0.0 \\
\hline$\%$ difference & $0.13 \%$ & $-0.23 \%$ & $0.0 \%$ \\
\hline
\end{tabular}




\section{M-MOF-274 Linker}

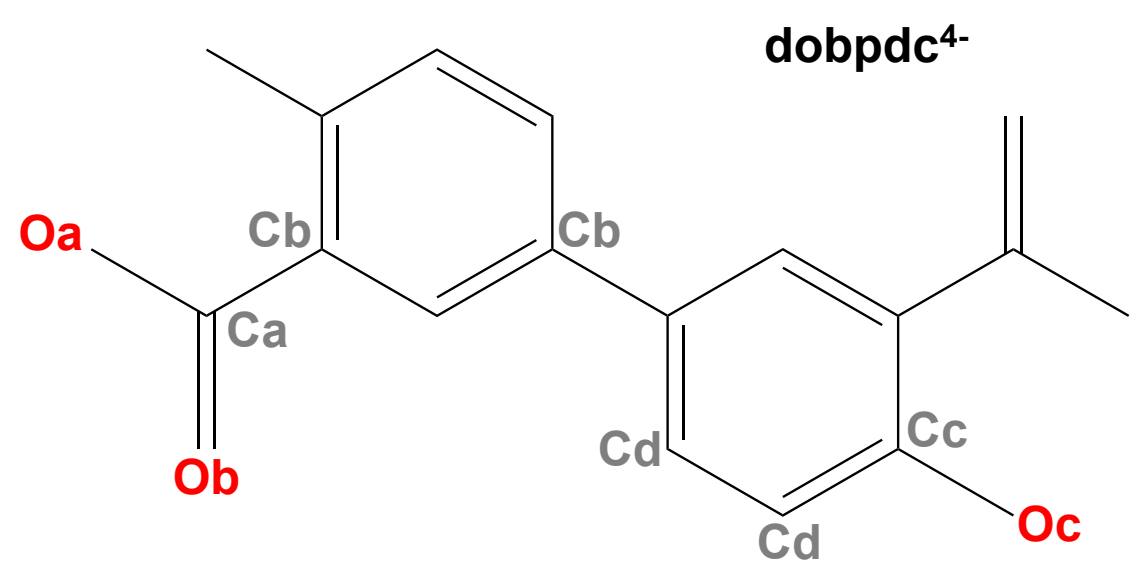

Supplementary Figure 13: The linker molecule in M-MOF-274, dobpdc $^{4-}=4,4$-dioxidobiphenyl-3,3-dicarboxylate, with atomic labels. Half of the atoms are labeled because the molecule is symmetrical. 


\section{Result Comparison from Different $m$-DFT Calculations}

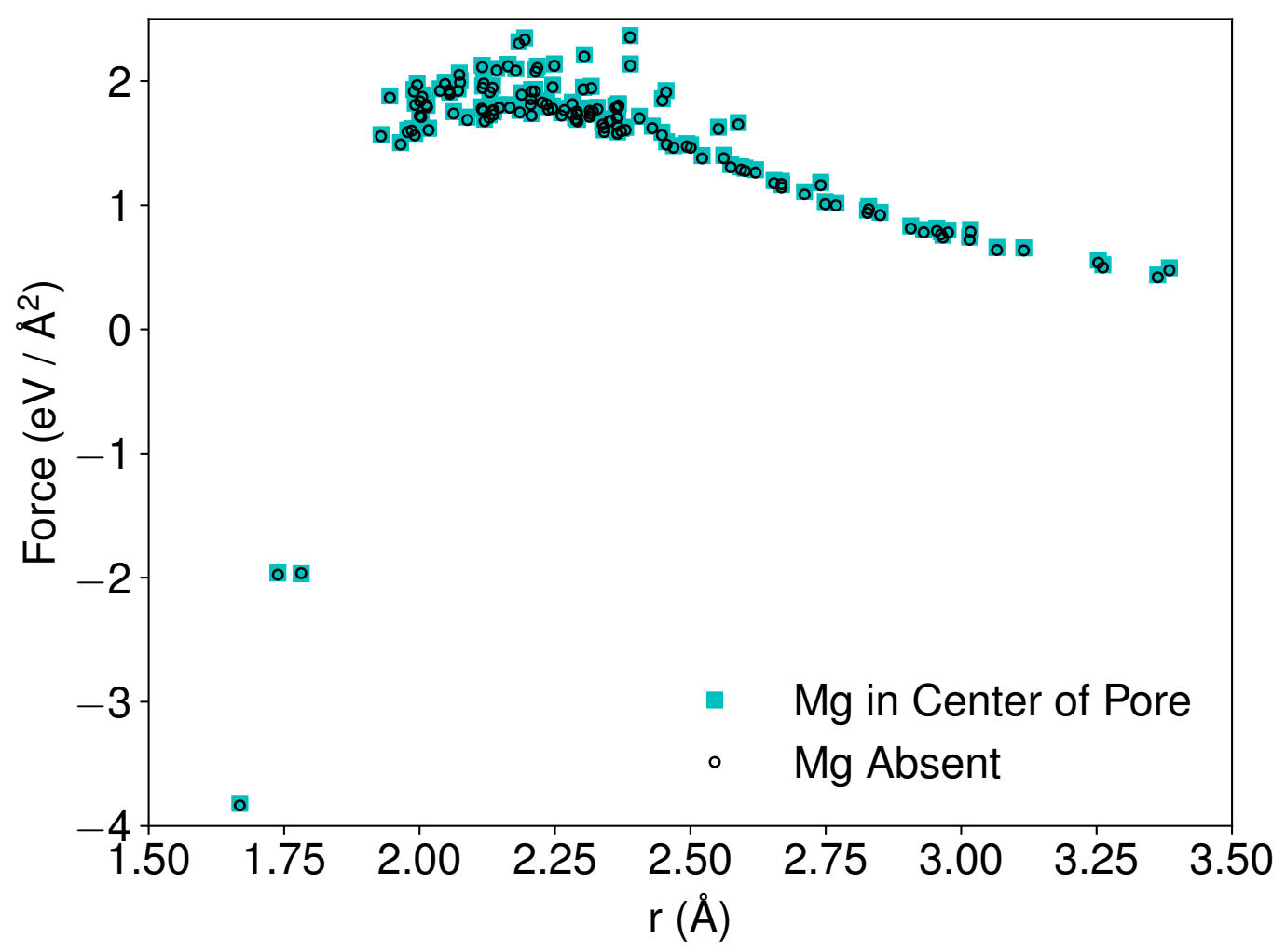

Supplementary Figure 14: Total Mg-Oa force as a function of distance estimated using the two types of $m$-DFT calculation: one where $\mathrm{Mg}$ is far removed from its original position in the structure, and one where it is absent altogether.

Figure 1 and 2, which are available as Supplementary Figures 14 and 15, show that the $\mathrm{Mg}$-Oa forces estimated using both types of $m$-DFT calculations are nearly identical. If the subtle electronic differences of the $m$-calculations are important for our parameterization, we would have expected to see a difference in the force estimations. From this we conclude that the electronic state deviations from the unperturbed framework do not manifest in the forces experienced by the oxygens coordinating to the target metal. 


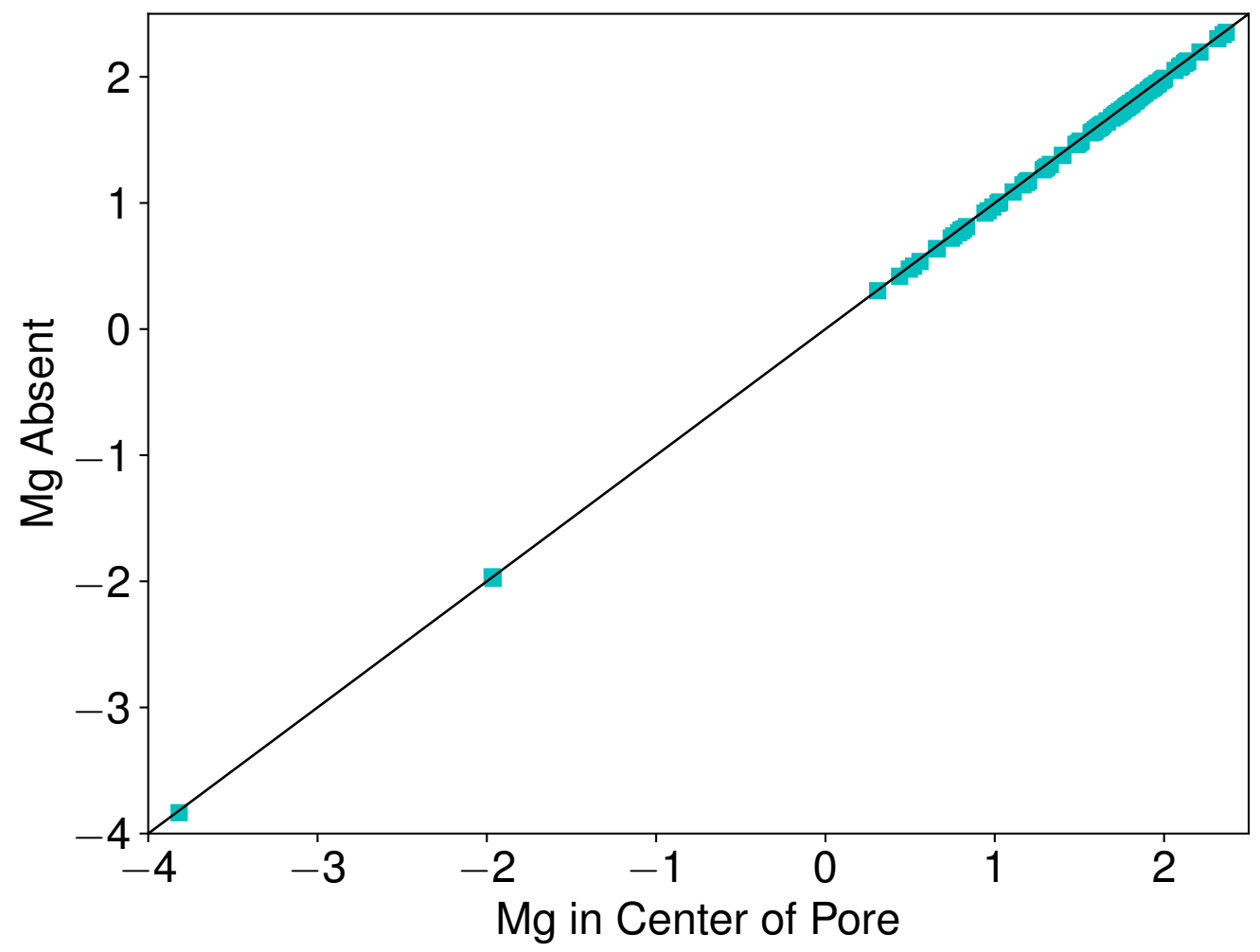

Supplementary Figure 15: Mg-Oa forces estimated using the two $m$-DFT calculations, plotted against each other. The diagonal line represents perfect agreement between the two sets of data. The maximum difference in estimated force for any configuration is $0.023 \mathrm{eV} / \AA$. 